\title{
COLOURIMETRIC CALIBRATION FOR PHOTOGRAPHY, PHOTOGRAMMETRY, AND PHOTOMODELLING WITHIN ARCHITECTURAL SURVEY
}

\author{
Laura Carnevali ${ }^{1}$, Fabio Lanfranchi ${ }^{1}$, Luca Martelli $^{1}$, Massimo Martelli ${ }^{2}$ \\ ${ }^{1}$ Dept. of History, Representation and Restoration of Architecture, "Sapienza" University of Rome, Italy, \\ laura.carnevali@uniroma1.it, fabio.lanfranchi@uniroma1.it, luca.martelli@uniroma1.it \\ ${ }^{2}$ Freelance computer programmer, massimo@ martel.li
}

KEY WORDS: Architecture, Colour Imaging, Survey, Colourimetric calibration, Photogrammetry, Photomodelling.

\begin{abstract}
:
In accordance with the "Declaration of Rome on architectural survey", we can affirm that recording and interpretation of colour information in photographic surveying, in photogrammetric surveying and in photomodelling requires careful planning of Colour Imaging processes. Information acquired by an optical sensor is influenced not just by the actual photographed scene, but also by the spectral sensitivity of the sensor. We have adopted, from the field of Cultural Heritage, a method of colourimetric calibration for digital photographs and have proposed some adjustments to finalise this process for the purposes of Architectural Survey. With the use of a colourimetric target and a non-linear transformation algorithm, our Colour Imaging method statistically reconstructs colours conventionally unrecordable by a commercial camera. In addition, this method reconstructs colours as if the photographed object were exposed to a standard illuminant, assessing a colour error parameter value for each photo. By including the colourimetric target in every shot and by applying the calibration algorithm to all photographs taken, the process correlates all data sets to a single standard illuminant: regarding photomodelling, this leads to a more uniform and detailed representation of the surfaces of virtual models. We present two successful examples of application: one focused on a design object with physioplastic decoration and another regarding a circular fountain in a historic villa.
\end{abstract}

\section{INTRODUCTION}

\subsection{General Instructions}

The more imaging (i.e. the processes of capturing, managing and displaying images) acquires relevance within Architectural Survey, the greater the role of colours treated by it in relation to knowledge of the investigated construction. Consequently, we have to take into account three issues:

A. During photographic and photogrammetric surveying, the volume of colour data which can be recorded is limited by camera spectral sensitivity. The risk of data loss could also arise when working on digital photos with inadequately calibrated monitors and printers, or with colour spaces too small for the needs. For the sake of production, management and preservation of documentation envisaged by the "Declaration of Rome on architectural survey", avoiding colour data loss in transitions from a digital device to another (or from a graphic application to another) is significant (Cundari, 2013).

B. Accuracy into recording and interpreting chromatic properties of a work of Architecture not only influences our knowledge about the surfaces of that construction: it also involves the detection of scale-invariant keypoints by algorithms dedicated to digital photogrammetry and photomodelling. Therefore, colour rendering influences individuation of homologous points between one photo and another. Scale-Invariant Feature Transform (SIFT), for example, is an essential algorithm for Structure from Motion (SfM) and indirectly acts as a function of the pixel colours making up the photograph. This because it is based on monochromatic intensities corresponding to these colours. In other words, SIFT relies on values obtained by converting the colour space of the photo into the grey scale (De Luca, 2011, Lowe, 2004).

C. In most cases, photographic and photogrammetric surveying involves outdoor shooting under uncontrolled conditions, where the features of natural illumination are complex and mutable, not to mention that any material captured in different places and at different times reacts to light in different ways (Gaiani et al., 2017).

Issue A had already been addressed in the field of Colour Imaging applied to Cultural Heritage (Apollonio et al., 2021, Melis et al., 2013). However, there is no critical review about implications of these issues within Architectural Survey at the moment: we discuss such outcomes in Section 2. Regarding issue $\mathrm{B}$ and $\mathrm{C}$, automated pre-processing methods for photogrammetry and photomodelling had already been proposed: these involve characterisation of an entire photographic data set by including a colourimetric target in one or two shoots (Apollonio et al., 2021, Gaiani et al., 2017, Versaci and Cardaci, 2011). In Section 3 we present an alternative to such methods, which allows us to evaluate colour fidelity in each single photo. In Section 4 we refer three applications of the proposed method and report their results. Finally, we draw some conclusions in Section 5.

\section{REVIEW OF LITERATURE}

\subsection{Colourimetry instruments}

2.1.1 Colour Matching Functions (CMF) and Chromaticity Diagram (CIE 1931): When light radiation is reflected by a surface and reaches the visual apparatus, it carries spectral emission information combined with information about spectral reflectance of that same surface. The human eye is unable to 
resolve the spectrum of received radiation with great precision. It rather performs coarse sampling through the sensitivity of retinal cones, encoding light radiation into three types of nervous stimuli. Considering only the visible light spectrum, cones sensitive to short waves transmit stimuli associated with blue; cones sensitive to medium waves transmit stimuli for green; cones sensitive to long waves transmit stimuli for red. In 1931, the Commission Internationale de l'Éclairage (CIE) ratified three weighting functions, based on the visual apparatus of a "standard observer": these are the Colour Matching Functions (CMF), which can be used to convert the parameters of a spectral radiation into a set of $\mathrm{X}, \mathrm{Y}, \mathrm{Z}$ values (Figure 1).

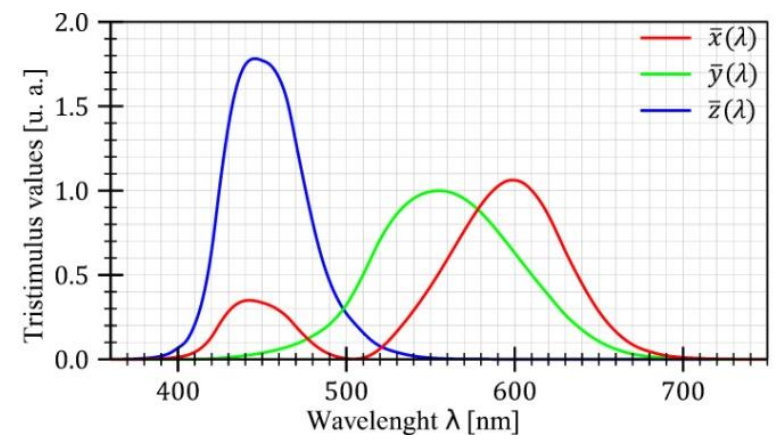

Figure 1. XYZ Colour Matching Functions (CMF) of CIE 1964 standard observer.

$\mathrm{X}, \mathrm{Y}$ and $\mathrm{Z}$ are the tristimulus coordinates of a radiation spectrum (within the visible light band). Therefore, in a space of coordinates $\mathrm{XYZ}$ a radiation spectrum can be represented with a point $\mathrm{Q}(\mathrm{X}, \mathrm{Y}, \mathrm{Z})$. Vector $\mathbf{O Q}$ intersects a plane passing through values $(001,010,100)$ on the axes, defining point $q$ with the following normalised coordinates (Figure 2):

$$
\begin{aligned}
& x=\frac{X}{X+Y+Z} \\
& y=\frac{Y}{X+Y+Z} \\
& z=\frac{Z}{X+Y+Z}=1-x-y
\end{aligned}
$$

where $x, y, z=$ chromaticity coordinates

$X, Y, Z=\mathrm{CIE} X Y Z$ tristimulus values

By varying the wavelength of a monochromatic source from far red to extreme blue, its chromaticity coordinates draw a counterclockwise arc inside a triangle with vertices $(001,010$, 100). The Chromaticity Diagram, also ratified by CIE in 1931, is obtained by connecting the ends of this arc with a segment in plane xy (Melis et al., 2013, Oleari, 2008, Oleari, 2016). The area thus circumscribed represents all colours perceptible to the average human eye, while points of maximum saturation are located on the outline (Figure 3). In order to determine the colourimetric coordinates of a measured colour, it is necessary to combine the spectral reflectance of that measurement, the CMF of the observer and a reference illuminant (Lindbloom, 2017).

2.1.2 Standard illuminants: The specification of colour measurements requires the specification of the illumination. Since 1931, CIE has also been ratifying "standard sources" and "standard illuminants": the term "illuminant" identifies the specification of a spectral power distribution which may or may not be physically realisable. In order to limit the number of illuminants used in colourimetry, CIE recommends using D65

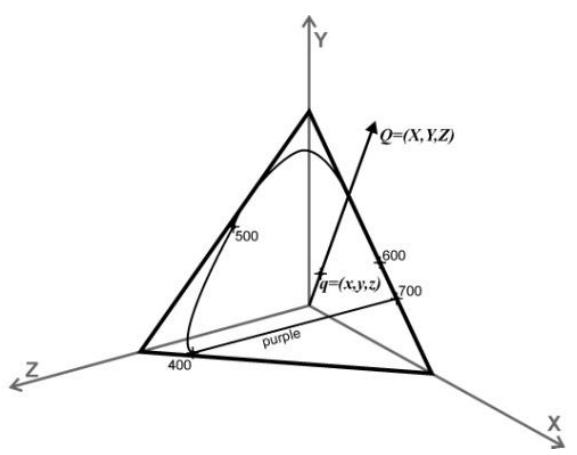

Figure 2. Normalised plane xyz in the tristimulus space.

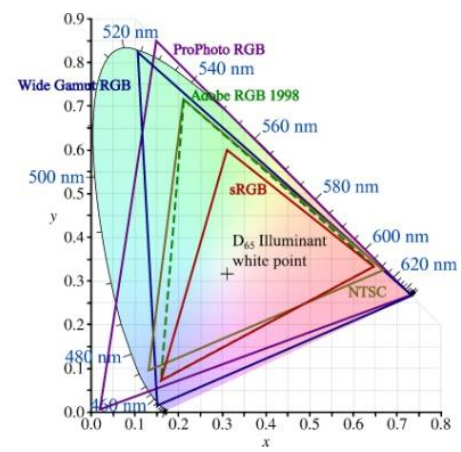

Figure 3. Comparison between gamuts of several colour spaces and CIE 1931 Chromaticity Diagram.

as the preferred standard illuminant: it has a correlated colour temperature of $6504 \mathrm{~K}$ and approximately represents the average daylight at midday from the northern sky, hence the name (Commission Internationale de l'Éclairage, 1999, Lee, 2005). D65 includes direct solar light and light diffused by a clear sky, so it is also known as the daylight standard illuminant. Illuminants have an impact on photographic images within Architectural Survey. If we want to take a photo of a construction with colour rendering as close as possible to the colours found under a given real illuminant (in a certain place, at a certain time and at a certain orientation), we could calibrate our equipment according to on site measurements executed by a spectrophotometer. However, those measures will only be valid for a scene that fulfils all the conditions of that specific situation: the likelihood of being able to reuse them depends on the accuracy level we require. In some cases, an hour is just enough for make us need new measurements with the spectrophotometer: colour information is very difficult to relate between two photos taken one hour apart. If, on the other hand, we want to obtain a colour rendering of the construction as if it were illuminated in a neutral environment and to have colour data comparable with information of other photos taken outside that precise context, we should calibrate our instrumentation according to a standard illuminant such as D65. This also applies when comparing colourimetric information from two sequences of photographs depicting the same construction under two noticeably different lighting conditions (e.g. photos taken at two significantly distant times in the same day).

\subsection{Colour Imaging technologies}

2.2.1 Silicon sensors, sensitivity and demosaicisation: In silicon sensors, the amount of electrical charge produced per photon, i.e. per wave, depends on the wavelength: they are typically responsive to wavelengths between a little over 300 
$\mathrm{nm}$ and a little over $1100 \mathrm{~nm}$. So, in order to be used in digital cameras, silicon sensors must be shielded by selective filters. Similarly to the cones of human retina, each silicon sensor model has its own specific sensitivity, which may differ from ones of other models. When we take a photo, the silicon sensor ponders the spectrum of emerging radiation according to its own sensitivity functions, then it saves all recorded data in a RAW file on a memory. What is contained in a RAW file is not a proper image, but the totality of all information that the camera recorded following settings we chose. Through a process of interpolation called demosaicisation, several raster files in different formats (JPG, BMP, TIFF, etc.) can be extracted from a single RAW file. For each sensor pixel, the other values of the two unrecorded primary colours can be interpolated from values of adjacent pixels: in this way, the three RGB channels are compiled and a complete matrix of colour data is assembled (Oleari, 2016).

2.2.2 Colour spaces and gamuts: From the point of view of Information Theory, synthesising a continuous spectrum with just three values means losing much of the original data: there can be no biunivocal relationship between a spectrum and colourimetric coordinates. While a spectrum corresponds to a specific set of colourimetric values, a set of colourimetric values can simultaneously relate to a large number of diverse spectra. This phenomenon, known as metamerism, physiologically involves the human eye. As a matter of fact, all manmade imaging relies on metamerism, which is the simulation of almost all colours by mixing few resources with fixed radiation spectra, or pigments with fixed spectral reflectances. Current CMF do not minimise metamerism and fail to appropriately represent a source at maximum saturation (monochrome), except for some portions of plane xy. The ideal CMF graphs would be linear and subtend triangular areas: such functions would correspond to a hypothetical Chromaticity Diagram shaped like an equilateral triangle (Melis et al., 2013). In spite of its imperfections, CIE 1931 (two-dimensionally represented by the Chromaticity Diagram in Figure 3) is the most common example of a colour space covering all colours perceivable by a standard observer. More generally, a colour space is the combination of a colour model and an appropriate mapping function for that model. Many colour spaces can be considered as subsets of CIE 1931, which is unused in practical applications because of its complexities. The domains or, in jargon, "gamuts" of the various colour spaces introduced over the years can be represented as triangles fully or partially contained by the Chromaticity Diagram in plane xy. Technological advances have enabled codification of ever-wider colour spaces: for example, Wide Gamut RGB colour space is more extensive than Adobe RGB 1998, which in turn is larger than sRGB (Figure 3). Similarly to what has been said about silicon sensor inputs, metamerism and various fabrication issues prevent development of colour spaces and graphic representation devices having the theoretical triangular Chromaticity Diagram as their gamut. Technological progress in Colour Imaging has, however, led to the introduction of graphic output devices (screens, printers, etc.) with increasingly extensive gamuts: the correct use of this instrumentation, however, requires appropriate colourimetric calibrations and the deployment of colour management processes.

2.2.3 Colour management and International Colour Consortium (ICC) profiles: If two colour spaces to be connected have different extensions, colourimetric data may be altered during transition from the source colour space to the destination colour space. Colour Management Systems (CMS) allow non-destructive adaptation of colourimetric data by assigning each device (camera, scanner, screen, printer, etc.) a colour profile, based on the characteristics of the machine itself. Colour profiles follow regulations and standards promoted by the International Colour Consortium (ICC): therefore, they are also known as ICC profiles. Device manufacturers and users can generate ICC profiles using specific instruments for colourimetric calibration (tristimulus colourimeters and spectrophotometers). An ICC profile contains conversion rules between the colour space values described in the profile and another standard colour space, called Profile Connection Space (PCS). According to ICC regulations, a PCS must be based on $\mathrm{L}^{*} \mathrm{a}$ *b system or CIE XYZ system (with standard illuminant D50). We can express these transformations using a correspondence table (interpolating whenever needed), mathematical formulas, or a combination of the two aforementioned methods. In the light of the above, it is clear that unplanned colour management and unreasonable application of ICC profiles can undermine photographic material destined to the operational archives of a survey. Therefore, we believe it is very useful to include planning of colour management and of ICC profiles into the contract specifications envisaged by the "Declaration of Rome on architectural survey" (Cundari, 2013).

\subsection{Colour Imaging workflows for Architectural and Cultural Heritage}

Processes for colourimetric calibration of digital still cameras and images derived therefrom are codified in ISO 17321: among the approaches described therein, one of the most widely used in Architectural and Cultural Heritage makes use of a colourimetric target (ISO/TC 42 Photography, 2012). With this technique, spectral values of the target under on site illuminantcan be related to spectral values of the same target under an illuminant obtained in laboratory, under controlled conditions: it allows colourimetric reconstruction of photo data as if the scene depicted had been subjected to such a controlled illuminant. During demosaicisation of each photograph, the colourimetric characterisation algorithm evaluates deviation between spectral data of the target in the photo and spectral data of the target under the controlled illuminant, expressing a value of colour error parameter $\Delta \mathrm{E}$ : the lower the $\Delta \mathrm{E}$ value, the closer colour rendering will be to the sample (Oleari, 2016). As far as instrumental surveying is concerned, most methods proposed in literature follow the practice of calibrating acquisition devices just once at the beginning of survey operations. In case of survey with a terrestrial laser scanner equipped with a camera, the target is placed on a spot of the site: only part of the scan captures the colourimetric target (Versaci and Cardaci, 2011). In case of photogrammetric surveying, the target is included once or twice maximum per acquired photo sequence (Apollonio et al., 2021, Gaiani et al., 2017). In these applications, the trend is to make colourimetric characterisation of images as automated as possible, using commercial targets and modifying or supplementing the accompanying preset software. Colourimetric calibration is thus encapsulated in an automatic phase of image pre-processing and compilation of the observed $\Delta \mathrm{E}$ values. Within Colour Imaging dedicated to Cultural Heritage, however, the most precise methods involve design and manufacture of custom-made colourimetric targets, as well as laboratory sampling of the spectral values of these targets under several standard illuminants (Melis et al., 2013). Although a process involving reduced use of colourimetric targets and the most possible automation offers indisputable advantages in terms of image processing, we thought appropriate to propose an alternative method, derived from the aforementioned advanced Cultural Heritage techniques and finalised at 
photomodelling: it features greater accuracy and takes into account on site illuminant variations between individual photographic shots.

\section{METHODOLOGY}

3.1 Assembly of a custom-made colourimetric target and sampling of its spectral reflection values

We assembled two colourimetric targets of different generations, according to the most advanced methods of Colour Imaging for Cultural Heritage: we equipped them with fiducial markers, useful for optional automatic detection during photo characterisation (Figure 4). We then subjected the target tiles to various standard illuminants in laboratory, including D65, and sampled their spectral reflectance values using a spectrophotometer. Thereafter, we entered these values into Visipick $^{\oplus}$ software for colourimetric calibration, created by ProfilocoloreSrl.

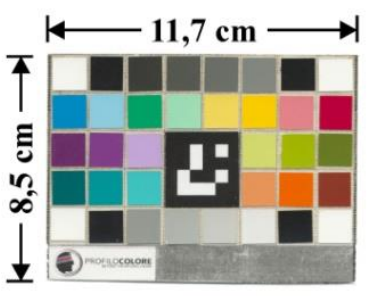

Number of tiles: 36

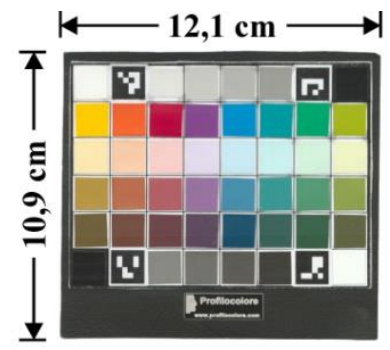

Number of tiles: 44
Figure 4. Colourimetric targets by Profilocolore Srl: left, Mark.06; right, Mark.07.

\subsection{On site shooting with colourimetric target}

In order to apply a SfM photomodelling process, we need to survey the object by taking reference measurements, by carefully planning Ground Sampling Distance (GSD) and coverages between photos, by materialising characteristic points and, finally, by taking different shots of the object surface. For each case study we just used one colourimetric target model, which we included in every single shot. Colourimetric calibration processes require the target illumination conditions to be as close as possible to those of the photographed subject. Ideally, the target should be oriented perpendicular to the optical axis, but it can be captured at moderately different positions as long as each of its tiles imprints at least a $10 \mathrm{px} \times$ $10 \mathrm{px}$ square on the photo: this rule also applies in relation to camera-to-target distance. To take full advantage of the camera sensitivity (an Olympus EM-10 $0^{\circ}$ ), we set its RAW file saving options to Adobe RGB colour space.

\subsection{Statistical recreation of colours through characterisation during demosaicisation}

To demonstrate the impact of colourimetric calibration and ICC profile choice on operational archives for photomodelling, we performed two parallel and independent demosaicisation processes on the RAW file data sets of each case study: one with RawTherapee software, producing rasters with TIFF format, 16-bit gamma integer precision, and destination ICC profile RTv4_sRGB (derived from sRGB colour space); the other with Visipick $^{\odot}$, generating rasters with TIFF format, 16bit gamma integer precision, but destination ICC profile Wide Gamut RGB. Stemming from RAW files expressed in Adobe
RGB colour space and spectral reflectance values sampled in laboratory under illuminant $\mathrm{D}_{65}$, Visipick ${ }^{\odot}$ performed non-linear colourimetric transformations based on CIE 64 CMF (Oleari, 2016): the algorithm statistically reconstructed all those colours present in Wide Gamut RGB colour space but excluded from Adobe RGB colour space. As stated in literature, non-linear colour characterisation processes require RGB values to be scaled when there is a change in exposure, but this may result in angular shifts in XYZ vectors, leading to visible colour shifts (Gaiani et al., 2017). However, in the advanced techniques for Cultural Heritage to which we referred, non-linear transformations are preferred as they allow corrections introducible by the chosen illuminant, the camera sensitivity or a combination of these two. In addition, the issue of angular shifts in XYZ vectors had been effectively contained by equipping our colourimetric targets with tiles specifically chosen to form certain colour scales, unlike those in the majority of commercially available kits, which averagely include fewer shades. Referencing to spectral reflectance values under $\mathrm{D}_{65}$, Visipick ${ }^{\odot}$ expressed a $\Delta \mathrm{E}_{00}$ value for the colour rendering of each demosaicised photo: $\Delta \mathrm{E}_{00}$ is expressed according to CIE 2000 Formula, which could not be transcribed here but is in public domain (Lindbloom, 2017, Oleari, 2016).

\subsection{Photomodelling with Structure from Motion technique}

In each of the first two case studies, a calibrated data set and an uncalibrated data set have been introduced into Agisoft Metashape $^{\odot}$ software, resulting in two separate virtual models. To prevent the presence of colourimetric targets and their supports from interfering with photo processing, we excluded them by applying a mask on each photograph. As stated in its User Manual, Agisoft Metashape ${ }^{\odot}$ does not alter colour data of photos during processing (Agisoft LCC, 2021): therefore, we deduced that colourimetric characteristics of photos calibrated by Visipick ${ }^{\odot}$ had been preserved in the textures of related virtual models. For the first two case studies, in addition to a direct comparison between the three-dimensional model from the calibrated data set and the one derived from the uncalibrated data set, we also performed a reliability comparison between the two associated dense point clouds.

\section{EXPERIMENTS AND RESULTS}

\subsection{Study Case No. 1: a decorated vase}

To easily ascertain the possibilities of the method described in Section 3, we conducted an initial test at the scale of design objects. We chose a subject that would immediately test the process effectiveness: a vase with a painted silicone physioplastic decoration (Figure 5). We conducted the whole experiment on a roof terrace on an afternoon with uniformly cloudy skies: this situation was certainly advantageous for a photographic survey under semi-controlled conditions and aimed at photomodelling. In order to materialise scalar reference points, we preferred to use a grid of paper fiducial markers on the tabletop rather than highlight characteristic points with pigments or pins, as these would inevitably cover part of the vessel surface, making it impossible to capture. We took a total of 78 photos in 5 circular paths at various heights and orientations around the object, including Mark.06 target (Figure 4) in each shot. In addition to obvious differences in colour rendering, all photos calibrated by Visipick ${ }^{\odot}$ have less noise and more sharpness, while the reliability of the associated dense point cloud is slightly better in some parts (Figure 6). 
Every $\Delta \mathrm{E}_{00}$ value obtained during the characterisation of each frame is shown in Table 1.
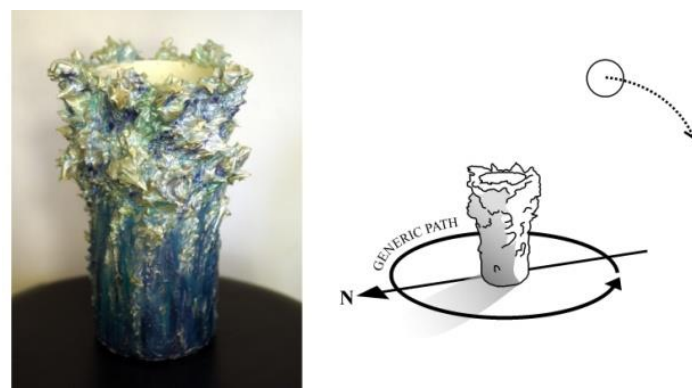

Figure 5. Left, the surveyed vessel; right, orientation of capture sequences.

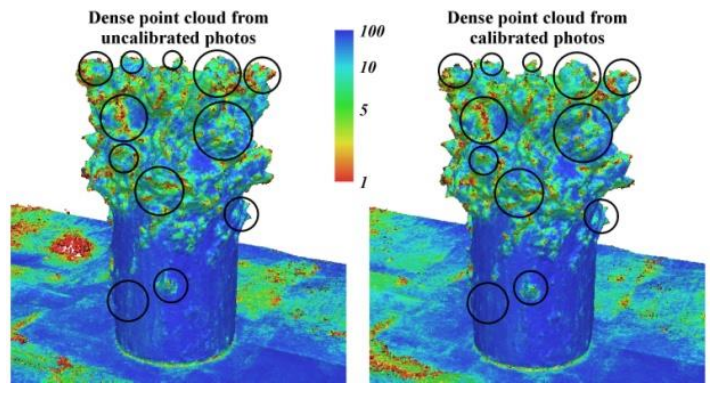

Figure 6. Reliability comparison between the dense point clouds of the vase. Improved parts are circled in black.
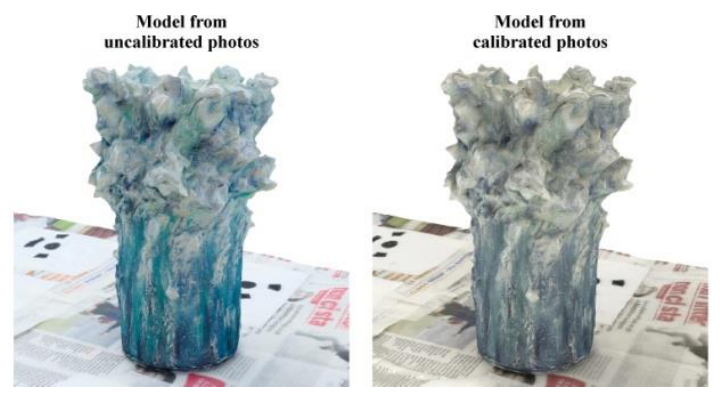

Figure 7. Comparison between the textured three-dimensional models of the vase.

\subsection{Study Case No. 2: a circular fountain}

To easily ascertain the possibilities of the method described in Section 3, we conducted an initial test at the scale of design objects. We chose a subject that would immediately test the process effectiveness: a vase with a painted silicone physioplastic decoration (Figure 5). We conducted the whole experiment on a roof terrace on an afternoon with uniformly cloudy skies: this situation was certainly advantageous for a photographic survey under semi-controlled conditions and aimed at photomodelling. In order to materialise scalar reference points, we preferred to use a grid of paper fiducial markers on the tabletop rather than highlight characteristic points with pigments or pins, as these would inevitably cover part of the vessel surface, making it impossible to capture. The purpose of the second test was to verify reproducibility of the method at architectural scale. We chose as our subject a nineteenth-century circular fountain located in the park of Villa Alberoni-Paganini in Rome (Figure 8). In order to have a scalar reference, we carried out a direct mensuration of the metal fence around the fountain. We took a total of 113 photos in 4 circular paths at various heights and locations around the object, including Mark.07 target in each shot (Figure 4). Considering that the photographic campaign was carried out under unfavourable and uncontrolled circumstances, as well as under two different lighting conditions, we can safely deem the results shown in Table 2 as satisfactory. Colourimetric calibration by Visipick $^{\odot}$ led to reconstruction of a more uniform illumination over the entire surface of the virtual model (Figure 10).
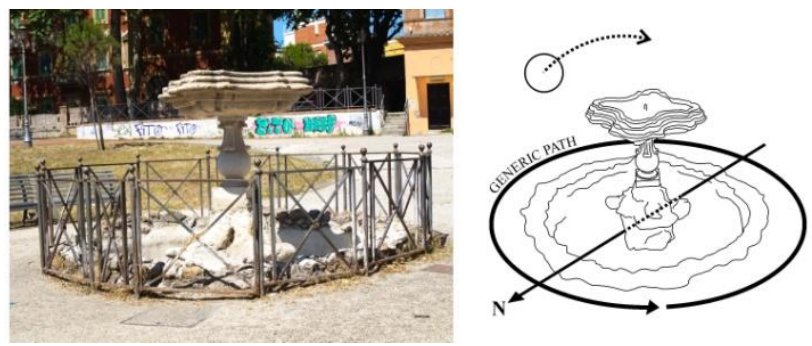

Figure 8. Left, the surveyed fountain in Villa AlberoniPaganini; right, orientation of capture sequences.

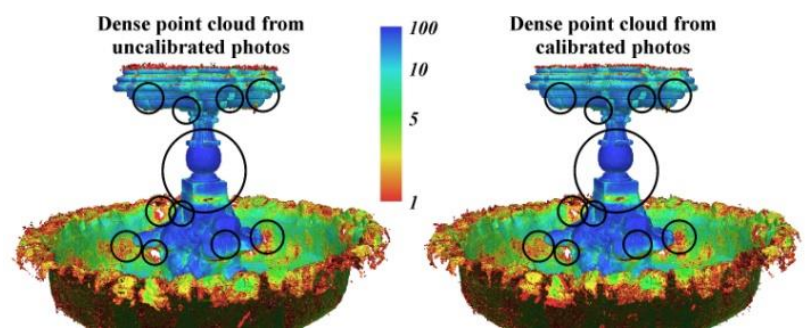

Figure 9. Reliability comparison between the dense point clouds of the fountain. Improved parts are circled in black.

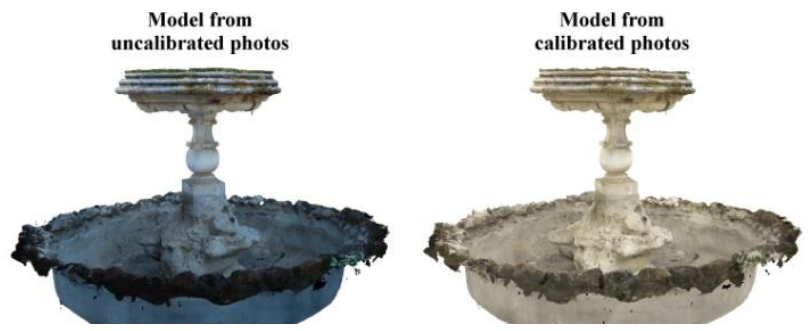

Figure 10. Comparison between the textured three-dimensional models of the fountain.

\subsection{Study Case No. 3: a historic aedicule}

In order to assess reproducibility of the method on even larger scale, we have attempted a photographic campaign of the Celebratory Aedicule of Villa Torlonia, also in Rome (Figure 11). Although the results shown in Table 3 have not proven overly problematic, we found that the chiaroscuro effects of such a complex architecture present difficulties: the presence of heavily shaded areas causes the colourimetric characterisation algorithm to overexpose surfaces which are already adequately lit, burning out some details. After a direct inspection of calibrated photos, we have nevertheless observed excellent sharpness in the previously shadowed parts, such that we can clearly distinguish brick joints (Figure 12). 


\begin{tabular}{|c|c|c|c|c|c|c|c|c|c|c|c|c|c|c|c|c|c|c|c|c|c|c|c|}
\hline 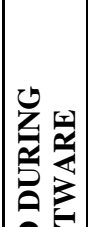 & $\underset{⿱ 乛}{\mathbb{a}}$ & 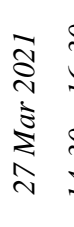 & 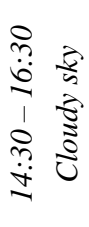 & $\begin{array}{l}\text { 合 } \\
\frac{0}{0} \\
\frac{a}{2}\end{array}$ & 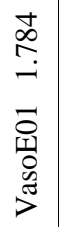 & 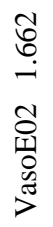 & 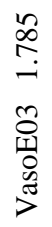 & 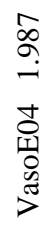 & 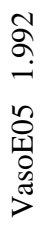 & 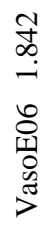 & 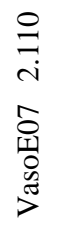 & 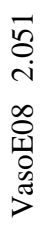 & 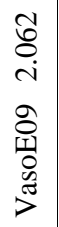 & $\begin{array}{l}2 \\
\stackrel{2}{i} \\
0 \\
0 \\
\text { II } \\
0 \\
0 \\
> \\
>\end{array}$ & 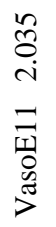 & 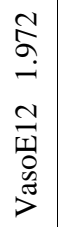 & 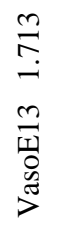 & 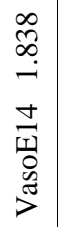 & & & & 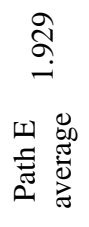 & \\
\hline 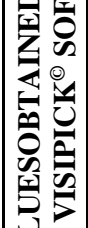 & $\underset{i}{a}$ & 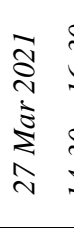 & 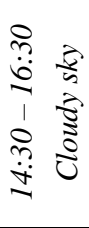 & 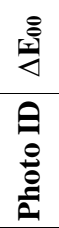 & 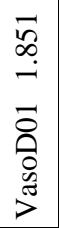 & 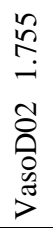 & 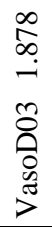 & 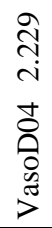 & 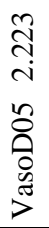 & 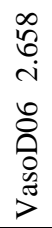 & 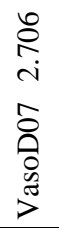 & 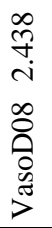 & 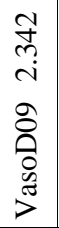 & $\begin{array}{l}m \\
\delta \\
i \\
0 \\
0 \\
0 \\
0 \\
\dot{z} \\
>\end{array}$ & 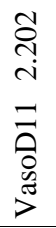 & 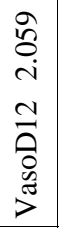 & 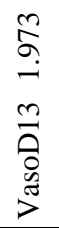 & 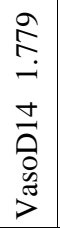 & 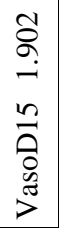 & $\begin{array}{l}\vec{n} \\
a \\
0 \\
0 \\
0 \\
0 \\
>\end{array}$ & & 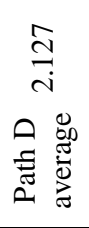 & \\
\hline 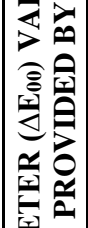 & $\underset{\Sigma}{u}$ & 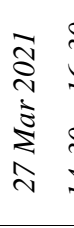 & 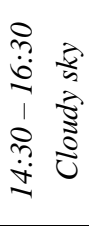 & 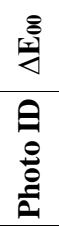 & 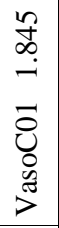 & 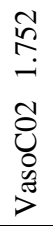 & 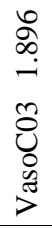 & 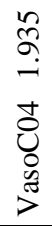 & 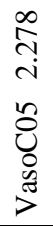 & 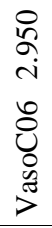 & 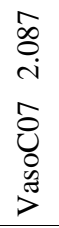 & 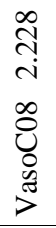 & $\begin{array}{l}8 \\
\dot{0} \\
i \\
o \\
\dot{0} \\
0 \\
o \\
\\
\end{array}$ & 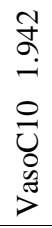 & 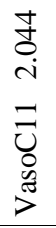 & 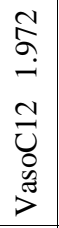 & 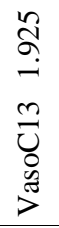 & 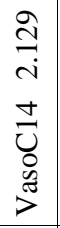 & 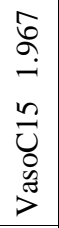 & & & 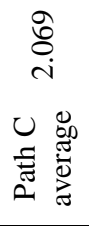 & \\
\hline 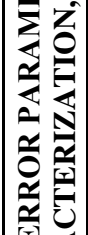 & $\underset{\Sigma}{\mathbb{Z}}$ & 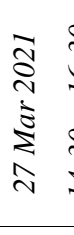 & 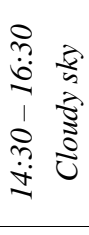 & 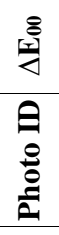 & $\begin{array}{l}\hat{\widehat{\sigma}} \\
- \\
\bar{\sigma} \\
0 \\
0 \\
\stackrel{\Xi}{>}\end{array}$ & 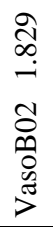 & 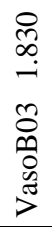 & 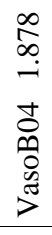 & 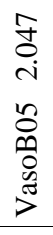 & 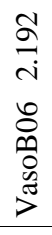 & 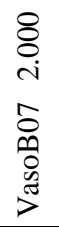 & $\begin{array}{l}\Xi \\
\Xi \\
i \\
\infty \\
0 \\
0 \\
0 \\
\vdots \\
> \\
>\end{array}$ & 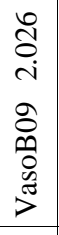 & $\begin{array}{l}m \\
\stackrel{1}{i} \\
0 \\
0 \\
0 \\
0 \\
\dot{0} \\
>\end{array}$ & 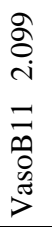 & 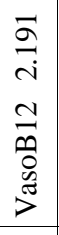 & 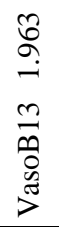 & 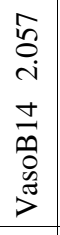 & $\begin{array}{l}\infty \\
\vdots \\
\vdots \\
n \\
\infty \\
0 \\
0 \\
> \\
>\end{array}$ & $\begin{array}{l}\infty \\
\infty \\
\infty \\
- \\
0 \\
\infty \\
0 \\
0 \\
0 \\
> \\
>\end{array}$ & 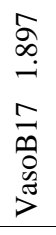 & 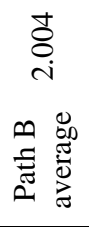 & 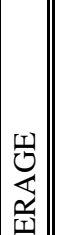 \\
\hline \begin{tabular}{|l}
0 \\
0 \\
0 \\
0
\end{tabular} & $\mid \underset{\Xi}{\mathbb{Z}}$ & 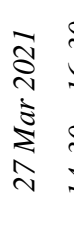 & 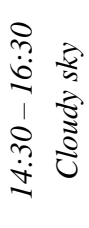 & 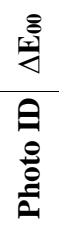 & 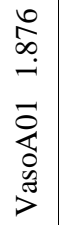 & 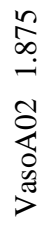 & 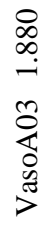 & 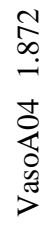 & 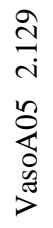 & $\begin{array}{l}\hat{o} \\
\text { in } \\
\text { i } \\
8 \\
0\end{array}$ & 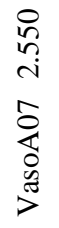 & $\begin{array}{l}0 \\
\& \\
i \\
i \\
\infty \\
\stackrel{0}{0} \\
0\end{array}$ & 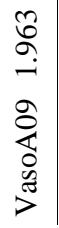 & 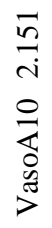 & 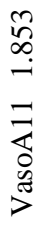 & 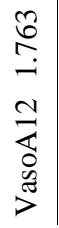 & 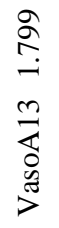 & 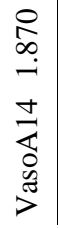 & 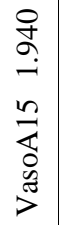 & 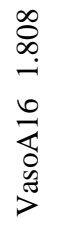 & & 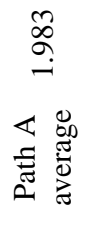 & 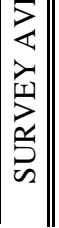 \\
\hline
\end{tabular}

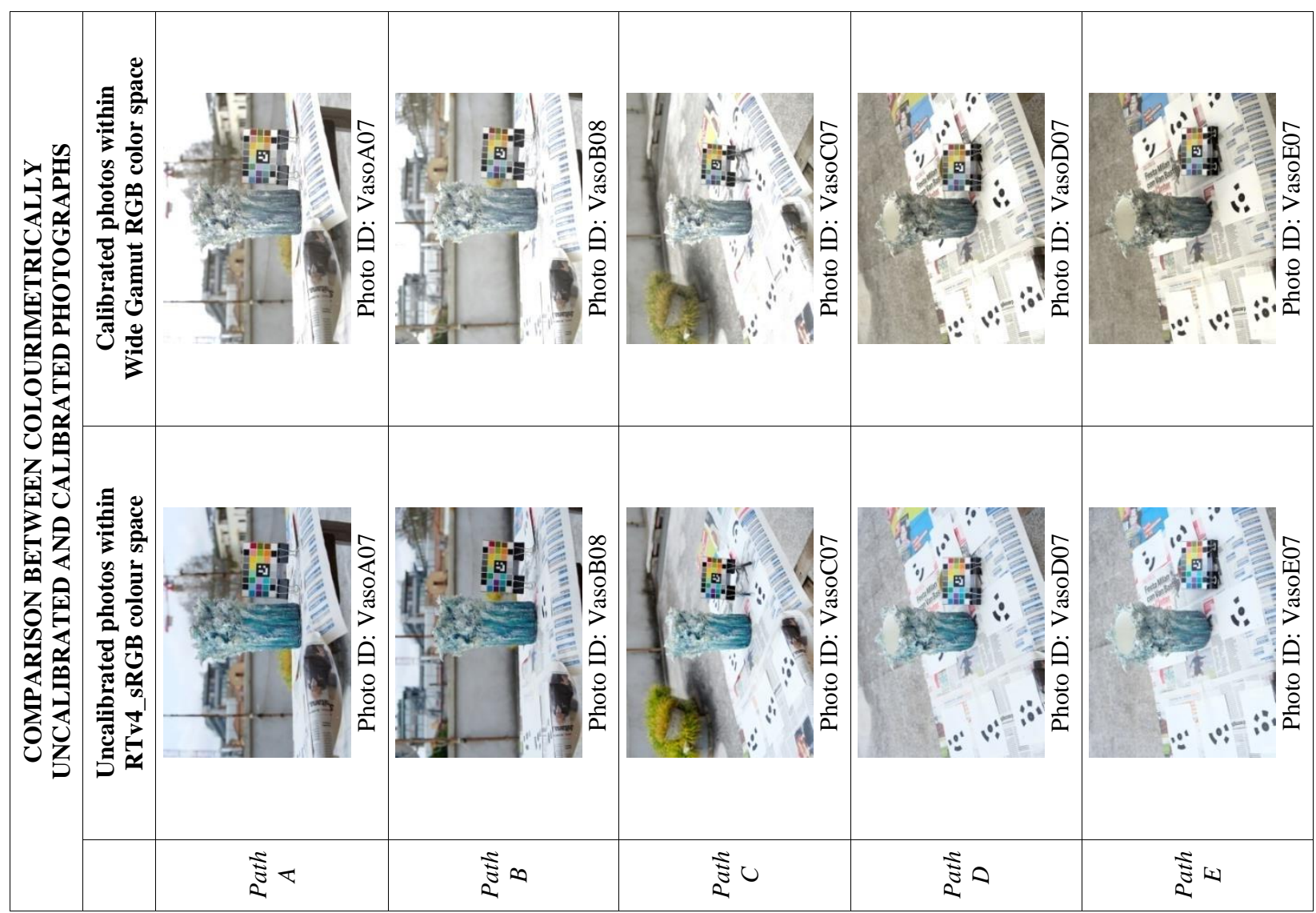

Table 1. Dataset of Study Case No. 1: the vase with physioplastic decoration in painted silicone. 

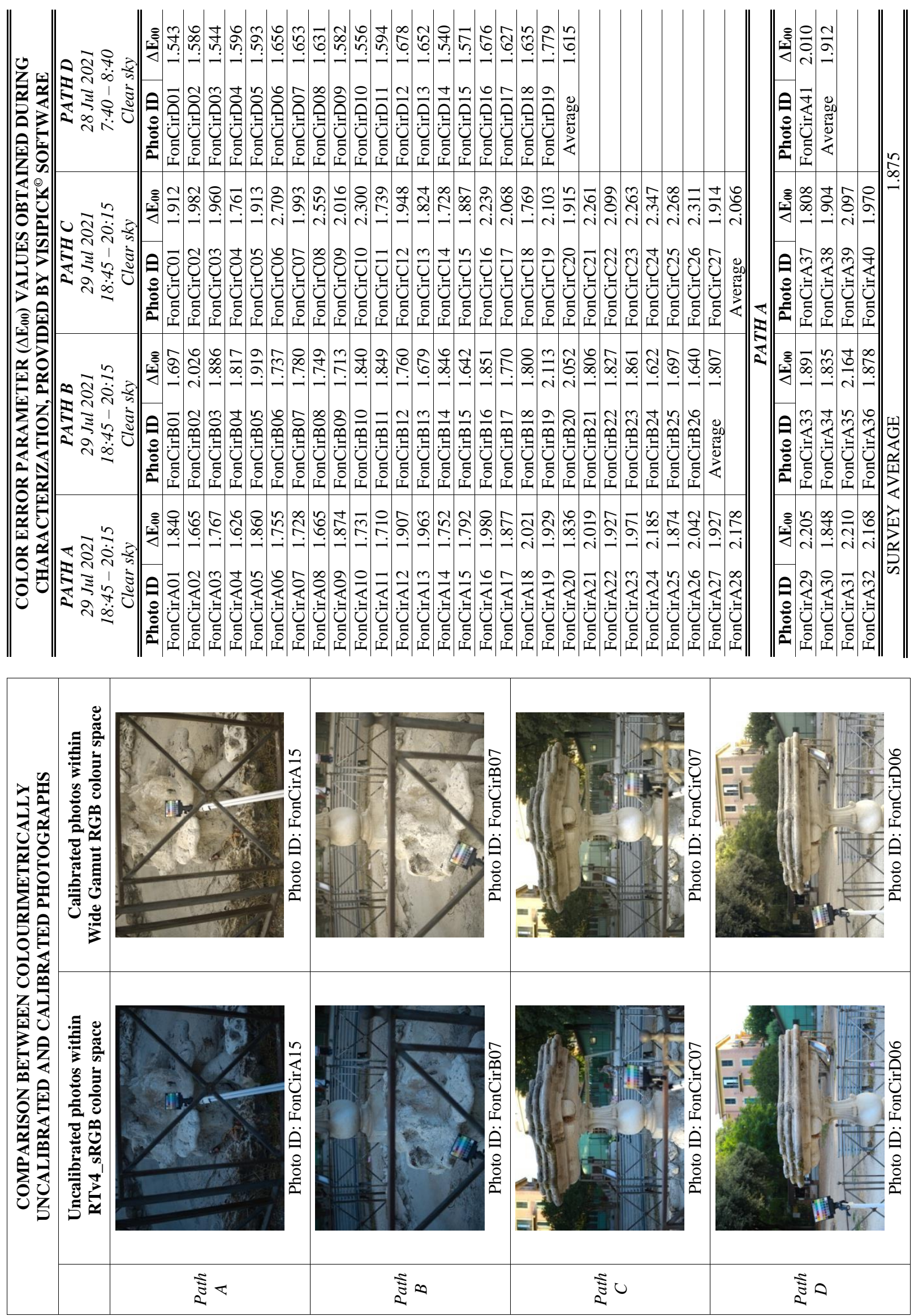

Table 2. Dataset of Study Case No. 2: the circular fountain in the park of Villa Alberoni-Paganini. 

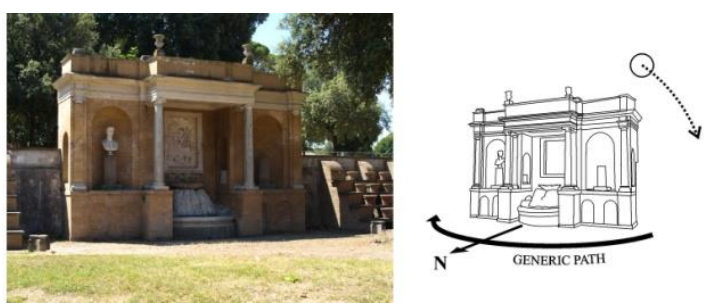

Figure 11. Left, the Celebratory Aedicule of Villa Torlonia; right, orientation of the capture sequences.

\begin{tabular}{|c|c|c|c|c|c|c|c|c|c|c|}
\hline ID & A01 & A02 & A03 & A04 & A05 & A06 & A07 & A08 & A09 & A10 \\
$\Delta \boldsymbol{E}_{\boldsymbol{0} \boldsymbol{0}}$ & 3.24 & 2.72 & 2.80 & 2.69 & 2.37 & 2.84 & 2.64 & 2.76 & 2.94 & 2.45 \\
\hline ID & A11 & A12 & A13 & A14 & A15 & A16 & A17 & A18 & A19 & A20 \\
$\Delta \boldsymbol{E}_{\boldsymbol{0} \boldsymbol{0}}$ & 2.55 & 2.49 & 2.62 & 3.41 & 2.68 & 2.46 & 2.78 & 2.36 & 2.34 & 2.47 \\
\hline ID & A21 & avg. & & & & & & & & \\
$\Delta \boldsymbol{E}_{\boldsymbol{0} \boldsymbol{0}}$ & 2.43 & 2.67 & & & & & & & & \\
\hline
\end{tabular}

Table 3. Colour error parameter $\left(\Delta \mathrm{E}_{00}\right)$ values for the Celebratory Aedicule in Study Case No. 3.

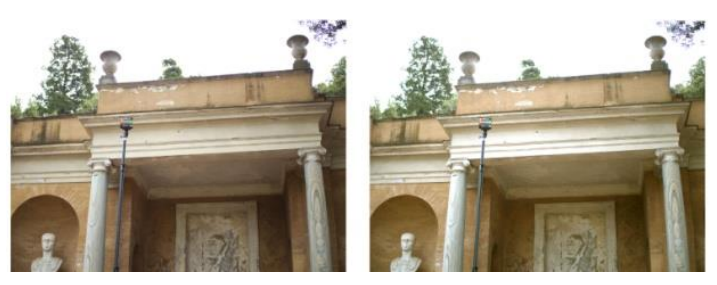

Figure 12. Two raster images from photo "A10": left, uncalibrated TIFF file; right, calibrated TIFF file.

\section{CONCLUSION}

In this work we have highlighted the importance of colour spaces and colour management in photographic and photogrammetric surveying, in photomodelling and, more generally, in Architectural Survey. We have illustrated a method for reconstructing, by colourimetric calibration, colour information unattainable from a conventionally used commercial camera, assessing the error of this statistical reconstruction in each photo. Finally, we have reported on our first applications of the proposed method in SfM photomodelling and have achieved more uniform illumination and more accurate reproduction of surfaces in the virtual models. Possible continuations of this research could concern greater interaction between colour management and digital three-dimensional models obtained by photomodelling, as well as resolution of some issues through the study of even more suitable colourimetric targets for Architectural Survey.

\section{CONFLICTS OF INTEREST}

The authors declare that they have no conflict of interest.

\section{ACKNOWLEDGEMENTS}

The authors would like to thank Profilocolore Srl for making their colourimetric targets and Visipick $^{\odot}$ software available for $^{\circ}$ the development of this paper.

\section{REFERENCES}

Agisoft LCC, 2021. Metashape Professional Edition ${ }^{\circledR}$ Software. Version 1.7.

Apollonio, F. I., Fantini, F., Garagnani, S., Gaiani, M., 2021. A Photogrammetry-Based Workflow for the Accurate 3D Construction and Visualization of Museums Assets. Remote Sensing, 13(3), 486. doi.org/10.3390/rs13030486.

Commission Internationale de l'Éclairage, 1999. CIE Standard Illuminants for Colorimetry (ISO 10526:1999/CIE S005/E1998).

Cundari, C., 2013. Il rilievo architettonico. Ragioni. Fondamenti. Applicazioni. Edizioni Kappa - Aracne Editrice S.r.l., Rome, Italy.

De Luca, L., 2011. La fotomodellazione architettonica. Rilievo, modellazione, rappresentazione di edifici a partire da fotografie. Dario Flaccovio Editore, Palermo, Italy.

Gaiani, M., Apollonio, F. I., Ballabeni, A., Remondino, F., 2017. Securing Color Fidelity in 3D Architectural Heritage Scenarios. Sensors, 17(11), 2437. doi.org/10.3390/s17112437.

ISO/TC 42 Photography, 2012. Graphic technology and photography - Colour characterisation of digital still cameras (DSCs) - Part 1: Stimuli, metrology and test procedures (ISO 17321-1:2012).

Lee, H., 2005. Introduction to Color Imaging Science. Cambridge University Press, Cambridge, UK.

Lindbloom, B., 2017. Computing xyz from spectral data (reflective and transmissive cases). http://www.brucelindbloom.com (30 July 2021).

Lowe, D. G., 2004. Distinctive Image Features from Scale Invariant Keypoints. Int. J. Comput. Vis., 60(2), 91-110. https://doi.org/10.1023/B:VISI.0000029664.99615.94.

Melis, M., Miccoli, M., Quarta, D., 2013. Multispectral Hypercolorimetry and automatic guided pigment identification: some masterpieces case studies. SPIE Proceedings Volume 8790,Optics for Arts, Architecture, and Archaeology IV, 87900W. doi.org/10.1117/12.2020643.

Oleari, C., 2008. Misurare il colore. Hoepli, Milan, Italy.

Oleari, C., 2016. Standard colorimetry. Definitions, Algorithms and Software. John Wiley and Sons Ltd, Chichester, UK.

Profilocolore Srl, 2021. Visipick @ Software, Version 1.3.

RawTherapee Development Team, 2020. RawTherapee Software, Version 5.8. rawtherapee.com (04 February 2020).

Versaci, A., Cardaci, A., 2011. Il rilievo "automatico" del colore: nuove tecnologie a supporto della lettura cromatica per il restauro dell'edilizia storica. Il colore nel costruito storico. innovazione, sperimentazione, applicazione, 13-15 October 2011, Lerici, Italy. https://openarchive.icomos.org/id/eprint/1203 (30 July 2021). 